\title{
CAESAREAN SCAR ECTOPIC: A RARE CASE
}

\begin{abstract}
Rumina Bagchi', Purvita Dam², Joydip Paul ${ }^{3}$, Sandip Chattopadhyay4, Sanjib Dutta ${ }^{5}$
${ }^{1}$ RMO Cum Clinical Tutor, Department of Gynaecology \& Obstetrics, College of Medicine \& Sagore Dutta Hospital. ${ }^{2}$ Associate Professor, Department of Gynaecology \& Obstetrics, College of Medicine \& Sagore Dutta Hospital. ${ }_{3}^{3}$ Assistant Professor, Department of Gynaecology \& Obstetrics, College of Medicine \& Sagore Dutta Hospital. ${ }^{4}$ RMO Cum Clinical Tutor, Department of Gynaecology \& Obstetrics, College of Medicine \& Sagore Dutta Hospital. ${ }^{5}$ Assistant Professor, Department of Gynaecology \& Obstetrics, College of Medicine \& Sagore Dutta Hospital.
\end{abstract}

\section{ABSTRACT}

Caesarean scar pregnancy is one of the rarest forms of ectopic pregnancies. But, with the increasing Caesarean section rates, its incidence is on the rise. Its diagnosis and treatment is a challenge specially as standard protocols are not available and treatment aims not only to spare the patient's life, but also to preserve her fertility.

The case reported here had initially been managed as an antenatal with intra-uterine pregnancy and had undergone an MTP. She presented with hypovolaemic shock and after resuscitation, was diagnosed as a patient with a Caesarean scar pregnancy. She underwent laparotomy with resection of the mass. Post-operative recovery was uneventful and she was discharged without complications.

\section{KEYWORDS}

Caesarean Scar Pregnancy.

HOW TO CITE THIS ARTICLE: Bagchi R, Dam P, Paul J, et al. Caesarean scar ectopic: a rare case. J. Evolution Med. Dent. Sci. 2016;5(48):3112-3115, DOI: $10.14260 /$ jemds/2016/723

\section{INTRODUCTION}

Caesarean scar ectopic is one which is implanted in the myometrium at the site of a previous Caesarean section scar.[1] It is considered to be the rarest form of ectopic pregnancy and a life-threatening condition. ${ }^{[2]}$ Because of the increasing number of Caesarean deliveries, its incidence has been rising ( 1 in 2000 normal pregnancies).[3] A recent case series estimates a rate of $0.15 \%$ Caesarean scar ectopic pregnancies in women with a previous Caesarean section and a rate of $6.1 \%$ of all ectopic pregnancies in women who had at least one previous Caesarean section. ${ }^{[4]}$ Complications may be life-threatening and impact negatively on future fertility. Thus, early diagnosis and management is of the utmost importance.

We describe here a case of Caesarean scar ectopic that presented with hypovolemic shock and was successfully treated by surgical intervention.

\section{CASE REPORT}

26 yr. old Mrs. Pinky Dey, $\mathrm{P}_{2+1}$, was admitted to our Institution, College of Medicine and Sagore Dutta Hospital, on 08.09.2015 in a state of shock through the Gynaecological Emergency. She had a history of severe bleeding per vaginum for 1 day. Her LMP was on 30.05.2015. On taking a detailed history, it was revealed that she had undergone an MTP 3 weeks back following which she had an episode of severe bleeding $\mathrm{p} / \mathrm{v}$ post-operatively for which she had received 2 units blood transfusion. She had been managed conservatively and subsequently discharged.

On examination, she was pale and in shock. Her pulse was feeble, rate being 120/min and BP 80/40 mmHg. Her abdomen was soft on palpation.

Financial or Other, Competing Interest: None.

Submission 30-04-2016, Peer Review 24-05-2016,

Acceptance 31-05-2016, Published 16-06-2016.

Corresponding Author:

Dr. Purvita Dam,

Flat 5H, Block 5, Lobby 1,

Avani Oxford, 136 Jessore Road,

Kolkata-700055.

E-mail: purvita_mdgo@yahoo.com

DOI: $10.14260 / \mathrm{jemds} / 2016 / 723$
Per speculum examination revealed profuse bleeding through the cervical os. Bimanual examination showed a bulky, soft uterus of 10 weeks size with a swelling of $3 \times 4 \mathrm{~cm}$, felt through the anterior fornix. A urine pregnancy test was done, which came to be faintly positive.

Shock was initially managed and resuscitation done with IV fluids. Investigations were sent, which also included blood sample for beta-HCG estimation and USG requisition was done.

Her $\mathrm{Hb}$ was $6.8 \mathrm{gm} \%$. A transabdominal USG showed a bulky uterus with a heterogeneous SOL $(4.5 \times 3.5 \mathrm{~cm})$ in the anterior wall of cervix with a simple hypoechoic cyst $(3.5 \mathrm{x}$ $3.1 \mathrm{~cm}$ ) on the left side. A probable diagnosis of cervical fibroid was given. TVS confirmed the TAS findings and in addition revealed an empty uterine cavity and cervical canal with echogenic collection around the cervix and the lower uterine segment. Beta-HCG was $46.24 \mathrm{mIU} / \mathrm{mL}$, which was above the non-pregnant level, but being low reduced the probabilities of a gestational trophoblastic disease.

Meanwhile, she received 3 units of blood transfusion. Based on our clinical suspicion, her urine pregnancy test result, beta-HCG report, we made a provisional diagnosis of a scar ectopic and she was put up for laparotomy. Abdomen was opened by low transverse incision. Uterus was delivered, bladder was found to be adherent to the anterior surface of the uterus, probably due to the previous 2 Caesarean sections. [Fig. 1].

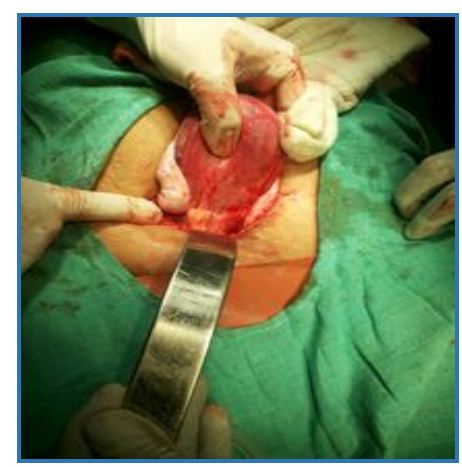

Fig. 1: Bladder Adherent to Lower Uterine Segment 
Bladder was pushed down gradually by sharp and blunt dissection to reveal the entire haemorrhagic mass [Fig. $2 \& 3$ ].

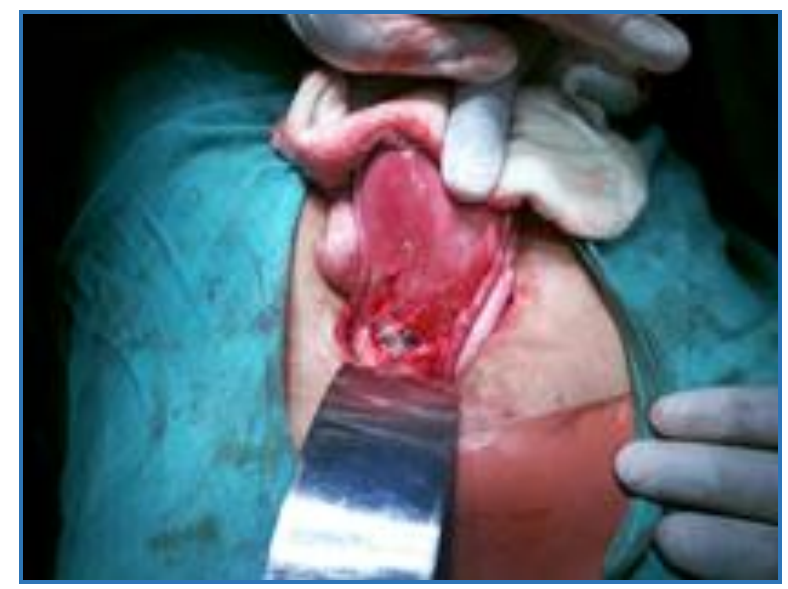

Fig. 2: Haemorrhagic Mass Visible

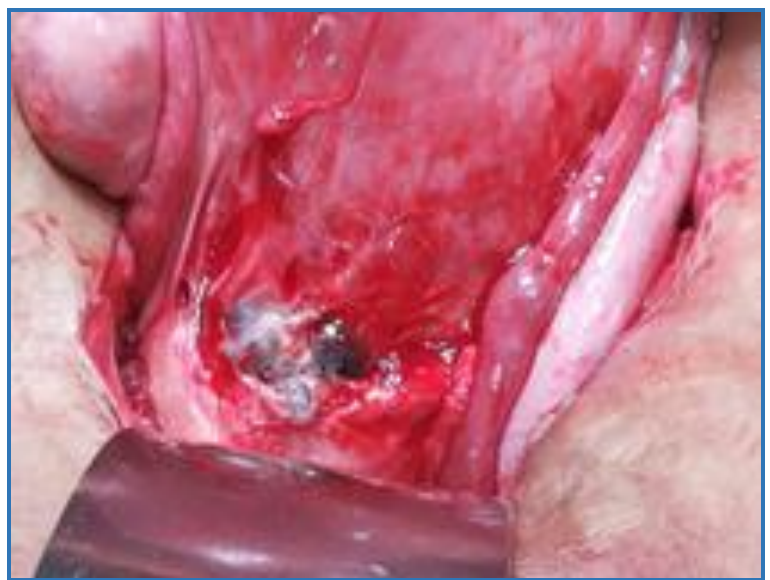

Fig. 3: Gradually Emerging Haemorrhagic Mass

As the bladder was further pushed down, it was observed that the mass occupied the entire region of the previous scars [Fig. 4].

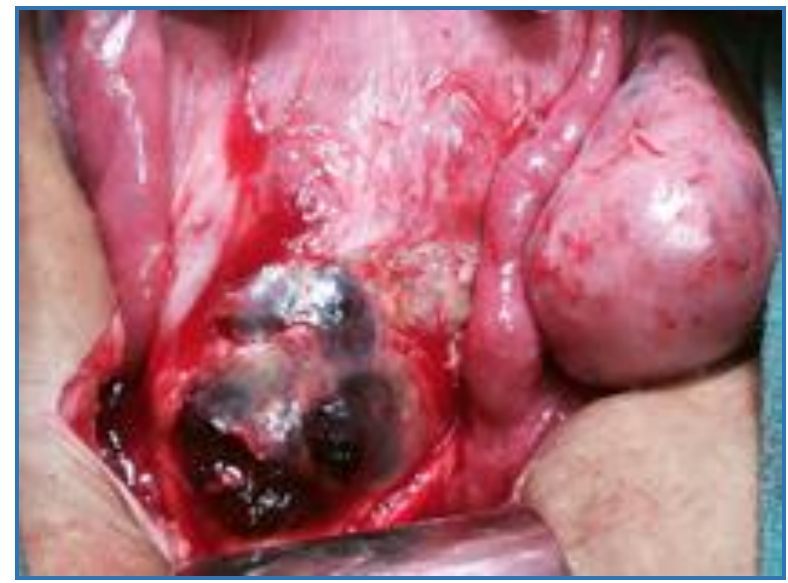

Fig. 4: The Mass Revealed in Entirety

Bilateral uterine artery was ligated, vasopressin injected all around the mass and it was scooped out in entirety [Fig. 5]. Once it was removed, the exposed fibrous margins were trimmed [Fig. 6] and the opening in the anterior uterine wall was repaired in 2 layers [Fig. 7].

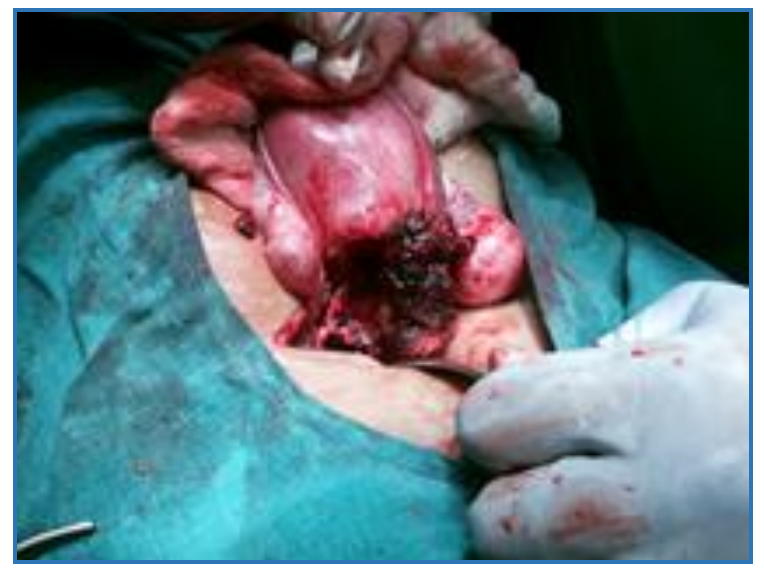

Fig. 5: Mass Scooped Out

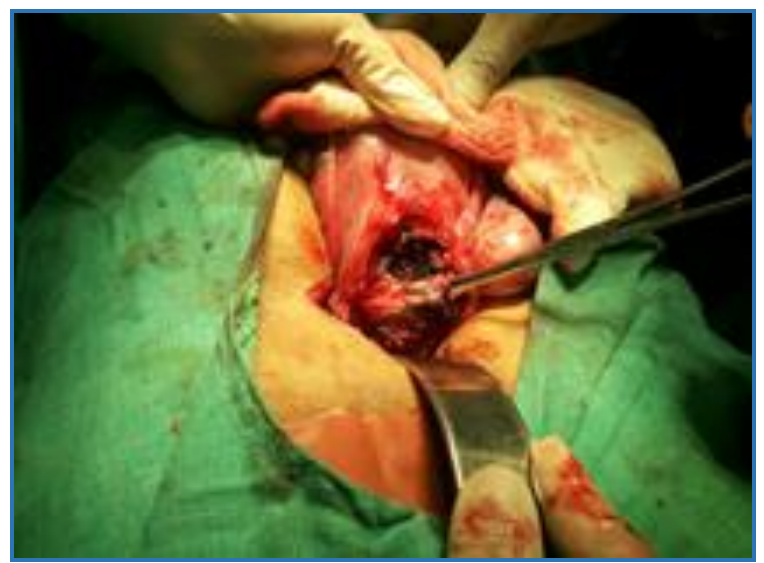

Fig. 6: Margins Trimmed

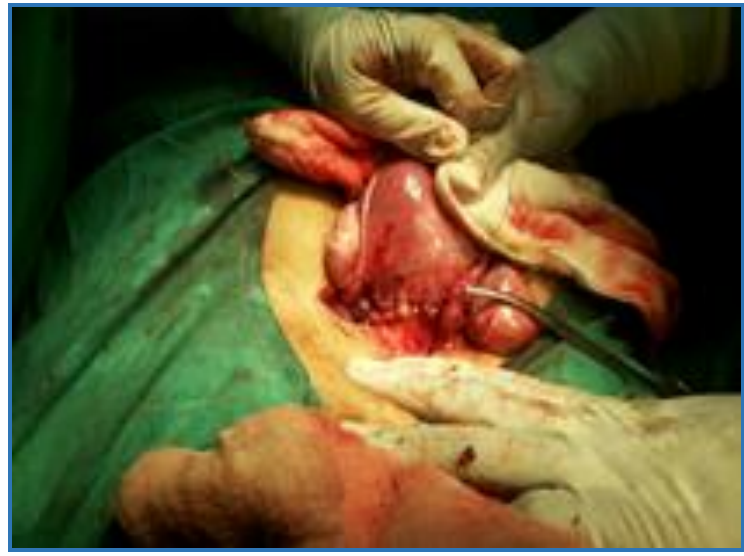

Fig. 7: Uterus Repaired in 2 Layers

Haemostasis was achieved. Judging her past obstetric history, her husband opted for bilateral tubal ligation. The removed mass was sent for HPE.

In the post-operative period, she was on antifibrinolytic agents and to prevent any AUB and to delay her next cycle progesterone was started. Catheter was removed on the $5^{\text {th }}$ post-operative day and she was discharged subsequently following an uneventful recovery.

Her HPE report came after 3 weeks, which showed many hyalinised villous structures amidst haemorrhage and necrotic material, a few cytotrophoblasts and intermediate trophoblasts. There was no evidence of malignancy. Histology was consistent with the clinical diagnosis of ectopic pregnancy. 


\section{DISCUSSION}

Caesarean scar ectopic is one of the rarest kind of pregnancies, but with the increasing number of Caesarean deliveries its incidence has been increasing to be about $1 / 2000$ normal pregnancies. [3]

The mechanism of implantation in this location is believed to be migration of the embryo through either the wedge defect in the lower uterine segment or a microscopic fistula within the scar. $[4,5,6]$

Such ectopics can be of two different types.[5], first being due to implantation of the gestational sac on the scar with progression towards either the cervico-isthmic space or towards the uterine cavity leading to a chance of a viable birth, but with an increased risk of life-threatening bleeding from the implantation site.[1] The second is a deep implantation into a post-caesarean defect with progression towards rupture and bleeding during the first trimester of pregnancy.

LSCS, adenomyosis, IVF, previous D \& C and manual removal of placenta are all risk factors. ${ }^{[5,6,7]}$ Our patient had a history of previous 2 Caesarean sections.

The clinical presentation ranges from vaginal bleeding with or without pain to ruptured uterus and hypovolaemic shock.[5,8,9] Many patients previously undiagnosed with caesarean scar pregnancy present with heavy bleeding $\mathrm{p} / \mathrm{v}$, haemoperitoneum and shock after trial of termination of an early pregnancy or D and C for a missed abortion.[10] In our case, patient had a similar presentation of heavy bleeding $\mathrm{p} / \mathrm{v}$ following trial of an early pregnancy termination.

Most of the cases which have been reported were diagnosed early in the $1^{\text {st }}$ trimester, gestational age at diagnosis ranging from $5^{\text {th }}$ to $12^{\text {th }}$ week.[11] Our patient presented at $10^{\text {th }}$ week of gestation.

The time interval from the last Caesarean section to the diagnosis of CSP ranged from 6 months to 12 years. ${ }^{[12]}$ In our case, the interval was 7 years.

Differential diagnosis includes cervical pregnancy and placenta accreta.[13]

The diagnosis may be made sonologically by visualising an enlarged scar with an embedded mass.[13,14] Typically, to differentiate from a cervical pregnancy, no myometrium between the gestational sac and bladder must be seen, because the gestational sac grows into the anterior portion of the isthmus.[15] To determine whether a CSP has occurred, USG in the sagittal position can be used to indicate a clear uterine cavity and an empty cervical canal.[16] In our case, TVS showed a bulky uterus with a SOL in the anterior wall of the lower part of the uterus and an empty uterine cavity and cervical canal.

Increased levels of beta-HCG aid in the diagnosis as was observed in our patient with a beta-HCG level of 46.24 microIU/mL.

The complications include rupture, haemorrhage, need for hysterectomy and increased maternal morbidity.

Management options for a CSP include medical or surgical intervention. Medical management includes methotrexate injection, locally or systemically or combined[17] and $\mathrm{KCl}$ injection. Surgical options are uterine artery embolization, excision of mass or hysterectomy. D \& C though done previously is now becoming obsolete.

A number of reports support the surgical alternative even in the presence of a non-bleeding patient.[2,5,18] This includes elective laparotomy and excision of the gestational mass, which was done in the present case. It is believed that the recurrence rate is reduced and follow-up time is shortened by this method. ${ }^{[19]}$

\section{CONCLUSION}

Despite its rarity, Caesarean scar ectopics contribute to significant morbidity and mortality of the patient. Every effort should be made to make an early, accurate diagnosis.

Its diagnosis and treatment is a challenge, especially as standard protocols are not available and treatment aims not only to spare the patient's life, but also to preserve her fertility.

It has always been a dictum to be ectopic-minded, but in this era of increasing rate of caesarean sections, we might go even a bit further and keep ourselves oriented to the possibility of scar ectopics in a post-LSCS patient.

\section{REFERENCES}

1. Herman A, Weinraub Z, Avrech O, et al. Follow up and outcome of isthmic pregnancy located in a previous caesarean section scar. British Journal of Obstetrics and Gynaecology 1995;102(10):839-41.

2. Fylstra DL, Pound-Chang T, Miller MG, et al. Ectopic pregnancy within a caesarean delivery scar: a case report. American Journal of Obstetrics and Gynaecology 2002;187(2):302-4.

3. Rotas MA, Haberman S, Levgur M. Caesarean scar ectopic pregnancies: aetiology, diagnosis, and management. Obstet Gynaecol 2006;107(6):1373-81.

4. Ash A, Smith A, Maxwell D. Caesarean scar pregnancy. BJOG 2007;114(3):253-63.

5. Vial Y, Petignat P, Hohlfeld P. Pregnancy in a caesarean scar. Ultrasound in Obstetrics and Gynaecology 2000;16(6):592-3.

6. Marchiolé P, Gorlero F, de Caro G, et al. Intramural pregnancy embedded in a previous caesarean section scar treated conservatively. Ultrasound in Obstetrics and Gynaecology 2004;23(3):307-9.

7. Lee CL, Wang CJ, Chao A, et al. Laparoscopic management of an ectopic pregnancy in a previous caesarean section scar. Human Reproduction 1999;14(5):1234-6.

8. Graesslin 0, Dedecker F, Quereux C, et al. Conservative treatment of ectopic pregnancy in a caesarean scar. Obstetrics and Gynaecology 2005;105(4):869-71.

9. Ginath S, Malinger G, Golan A, et al. Successful laparoscopic treatment of a ruptured primary abdominal pregnancy. Fertility and Sterility 2000;74(3):601-2.

10. Timor-Tritsch IE, Monteagudo A, Santos R, et al. The diagnosis, treatment and follow-up of caesarean scar pregnancy. Am J Obstet Gynaecol 2012;207(1):44.e1-13.

11. Seow KM, Huang LW, Lin YH, et al. Caesarean scar pregnancy: issues in management. Ultrasound in Obstetrics and Gynaecology 2004;23(3):247-53.

12. Nankali A, Ataee M, Shahlazadeh $H$, et al. Surgical management of the caesarean scar ectopic pregnancy: a case report. Case Reports in Obstetrics and Gynaecology Article ID 525187, 2013;2013:pages 3.

13. Ofili-Yebovi D, Ben-Nagi J, Sawyer E, et al. Deficient lowersegment caesarean section scars: prevalence and risk factors. Ultrasound in Obstetrics and Gynaecology 2008;31(1):72-7. 
14. Armstrong V, Hansen WF, Van Voorhis BJ, et al. Detection of caesarean scars by transvaginal ultrasound. Obstetrics and Gynaecology 2003;101(1):61-5.

15. Rizk B, Holiday CP, Owens S, et al. Cervical and caesarean scar ectopic pregnancies: diagnosis and management. Middle East Fertility Society Journal 2013;18(2):67-73.

16. Fylstra DL. Ectopic pregnancy not within the (distal) fallopian tube: aetiology, diagnosis, and treatment. Am J Obstet Gynaecol 2012;206(4):289-99.
17. Nawroth F, Foth D, Wilhelm L, et al. Conservative treatment of ectopic pregnancy in a caesarean section scar with methotrexate: a case report. Eur J Obstet Gynaecol 2001;99:135-7.

18. Seow KM, Hwang JL, Tsai YL. Ultrasound diagnosis of a pregnancy in a caesarean section scar. Ultrasound Obstet Gynaecol 2001;18(5):547-51.

19. Maymon R, Halperin R, Mendlovic S, et al. Ectopic pregnancies in caesarean section scars: the 8 year experience of one medical centre. Hum Reprod 2004;19(2):278-84. 